\title{
ELLIPTIC PROBLEMS WITH NONMONOTONE DISCONTINUITIES AT RESONANCE
}

\author{
HALIDIAS NIKOLAOS
}

Received 23 January 2002

Using the critical point theory of Chang (1981) for locally Lipschitz functionals, we prove an existence theorem for some elliptic problems at resonance with no Carathéodory forcing term.

\section{Introduction}

In this paper, we consider elliptic problems with discontinuities at resonance. Recently, Bouchala and Drábek [2] using an extended type of Landesman-Lazer conditions proved existence theorems for both coercive and noncoercive cases. They assumed that the nonlinear right-hand side is of Carathéodory type. Here, we are interested in this problem but we do not assume that the right-hand side is Carathéodory and moreover we seek for nontrivial solutions.

For the noncoercive case we obtain a nontrivial solution using the mountainpass theorem for locally Lipschitz functionals due to Chang [3]. The problem is an elliptic problem at resonance. Let $Z \subseteq \mathbb{R}^{N}$ be a bounded domain with a $C^{1}$-boundary $\Gamma$,

$$
\begin{gathered}
-\operatorname{div}\left(\|D x(z)\|^{p-2} D x(z)\right)-\lambda_{1}|x(z)|^{p-2} x(z)=f(z, x(z)) \quad \text { a.e. on } Z, \\
\left.x\right|_{\Gamma}=0 .
\end{gathered}
$$

In Section 2, we recall some facts and definitions from the critical point theory for locally Lipschitz functionals and the subdifferential of Clarke.

\section{Preliminaries}

Let $Y$ be a subset of $X$. A function $f: Y \rightarrow \mathbb{R}$ is said to satisfy a Lipschitz condition (on $Y$ ) provided that, for some nonnegative scalar $K$, we have

$$
|f(y)-f(x)| \leq K\|y-x\|
$$

Copyright (C) 2002 Hindawi Publishing Corporation Abstract and Applied Analysis 7:9 (2002) 497-507

2000 Mathematics Subject Classification: 35J25, 35J60

URL: http://dx.doi.org/10.1155/S1085337502203061 
for all points $x, y \in Y$. Let $f$ be Lipschitz near a given point $x$, and let $v$ be any other vector in $X$. The generalized directional derivative of $f$ at $x$ in the direction $v$, denoted by $f^{o}(x ; v)$, is defined as follows:

$$
f^{o}(x ; v)=\limsup _{\substack{y \rightarrow x \\ t \downarrow 0}} \frac{f(y+t v)-f(y)}{t},
$$

where $y$ is a vector in $X$ and $t$ is a positive scalar. If $f$ is Lipschitz of $\operatorname{rank} K$ near $x$, then the function $v \rightarrow f^{o}(x ; v)$ is finite, positively homogeneous, subadditive, and satisfies $\left|f^{o}(x ; v)\right| \leq K\|v\|$. In addition, $f^{o}$ satisfies $f^{o}(x ;-v)=(-f)^{o}(x ; v)$. Now we are ready to introduce the generalized gradient denoted by $\partial f(x)$,

$$
\partial f(x)=\left\{w \in X^{*}: f^{o}(x ; v) \geq\langle w, v\rangle \forall v \in X\right\} .
$$

Some basic properties of the generalized gradient of locally Lipschitz functionals are the following:

(a) $\partial f(x)$ is a nonempty, convex, weakly compact subset of $X^{*}$ and $\|w\|_{*} \leq$ $K$ for every $w$ in $\partial f(x)$;

(b) for every $v$ in $X$, we have

$$
f^{o}(x ; v)=\max \{\langle w, v\rangle: w \in \partial f(x)\} .
$$

If $f_{1}, f_{2}$ are locally Lipschitz functions, then

$$
\partial\left(f_{1}+f_{2}\right) \subseteq \partial f_{1}+\partial f_{2} .
$$

We recall the Palais-Smale (PS) condition introduced by Chang [3].

Definition 2.1. A Lipschitz function $f$ satisfies the Palais-Smale condition if any sequence $\left\{x_{n}\right\}$, along which $\left|f\left(x_{n}\right)\right|$ is bounded and

$$
\lambda\left(x_{n}\right)=\min _{w \in \partial f\left(x_{n}\right)}\|w\|_{X^{*}} \longrightarrow 0,
$$

possesses a convergent subsequence.

The PS-condition can also be formulated as follows (see Costa and Gonçalves [5]):

$(\mathrm{PS})_{\mathcal{c},+}^{*}$ : whenever $\left(x_{n}\right) \subseteq X,\left(\varepsilon_{n}\right),\left(\delta_{n}\right) \subseteq \mathbb{R}_{+}$are sequences with $\varepsilon_{n} \rightarrow 0, \delta_{n} \rightarrow 0$, and such that

$$
f\left(x_{n}\right) \longrightarrow c, \quad f\left(x_{n}\right) \leq f(x)+\varepsilon_{n}\left\|x-x_{n}\right\| \quad \text { if }\left\|x-x_{n}\right\| \leq \delta_{n},
$$

then $\left(x_{n}\right)$ possesses a convergent subsequence, $x_{n^{\prime}} \rightarrow \hat{x}$.

Similarly, we define the (PS) ${ }_{c}^{*}$ condition from below, (PS) $)_{c,-}^{*}$, by interchanging $x$ and $x_{n}$ in (2.7). And finally, we say that $f$ satisfies (PS) ${ }_{c}^{*}$ provided that it satisfies $(\mathrm{PS})_{c,+}^{*}$ and $(\mathrm{PS})_{c,-}^{*}$. 
Note that these two definitions are equivalent when $f$ is locally Lipschitz functional.

We mention some facts about the first eigenvalue of the $p$-Laplacian. Consider the first eigenvalue $\lambda_{1}$ of $\left(-\Delta_{p}, W_{o}^{1, p}(Z)\right)$. From Lindqvist [6] we know that $\lambda_{1}>0$ is isolated and simple, that is, any two solutions $u, v$ of

$$
\begin{gathered}
-\Delta_{p} u=-\operatorname{div}\left(\|D u\|^{p-2} D u\right)=\lambda_{1}|u|^{p-2} u \quad \text { a.e. on } Z, \\
\left.u\right|_{\Gamma}=0, \quad 2 \leq p<\infty
\end{gathered}
$$

satisfy $u=c v$ for some $c \in \mathbb{R}$. In addition, the $\lambda_{1}$-eigenfunctions do not change sign in $Z$. Finally, we have the following variational characterization of $\lambda_{1}$ (Rayleigh quotient):

$$
\lambda_{1}=\inf \left[\frac{\|D x\|_{p}^{p}}{\|x\|_{p}^{p}}: x \in W_{o}^{1, p}(Z), x \neq 0\right] .
$$

We are going to use the mountain-pass theorem of Chang [3].

Theorem 2.2. If a locally Lipschitz functional $f: X \rightarrow \mathbb{R}$ on the reflexive Banach space $X$ satisfies the PS-condition and the hypotheses,

(i) there exist positive constants $\rho$ and a such that

$$
f(u) \geq a \quad \forall x \in X \text { with }\|x\|=\rho
$$

(ii) $f(0)=0$ and there is a point $e \in X$ such that

$$
\|e\|>\rho, \quad f(e) \leq 0,
$$

then there exists a critical value $c \geq a$ of $f$ determined by

$$
c=\inf _{g \in G} \max _{t \in[0,1]} f(g(t)),
$$

where

$$
G=\{g \in C([0,1], X): g(0)=0, g(1)=e\} .
$$

Motreanu and Panagiotopoulos [7, Theorem 1 and Corollary 1] provide a proof for the generalized mountain-pass theorem for locally Lipschitz func tionals.

\section{Existence theorems}

Here, we give the hypotheses that we need for our existence theorem.

Let

$$
f_{1}(z, x)=\liminf _{x^{\prime} \rightarrow x} f\left(z, x^{\prime}\right), \quad f_{2}(z, x)=\limsup _{x^{\prime} \rightarrow x} f\left(z, x^{\prime}\right) .
$$


Hypothesis 3.1. The function $f: Z \times \mathbb{R} \rightarrow \mathbb{R}$ is an $N$ measurable function (i.e., if $x(z)$ is measurable so is $\left.f_{1}(x(z)), f_{2}(x(z))\right)$, and moreover,

(i) for almost all $z \in Z$ and all $x \in \mathbb{R},|f(z, x)| \leq c_{1}|x|^{p^{-1}}+c|x|^{p^{*}-1}$, with $p^{*}=N p /(N-p)$

(ii) there exist $\theta>p$ and $r_{o}>0$ such that for all $|x| \geq r_{o}$, and all $v \in\left[f_{1}(z, x)\right.$, $f_{2}(z, x)$ ] we have $0<\theta F(z, x) \leq v x$, and moreover there exists some $a_{1} \in$ $L^{1}(Z)$ such that $F(z, x) \geq c_{3}|x|^{\theta}-a_{1}(z)$ for every $x \in \mathbb{R}$, with $F(z, x)=$ $\int_{o}^{r} f(z, r) d r$

(iii) uniformly, for all $z \in Z$ we have $\limsup _{x \rightarrow 0}\left(p F(z, x) /|x|^{p}\right) \leq \theta(z) \leq 0$ with $\theta(z) \in L^{\infty}(Z)$ and $\theta(z)<0$ on a set of positive measure.

Remark 3.2. Hypothesis (iii) is the crucial one in order to have a nontrivial solution. Many authors have used such kind of hypothesis but this form is more general, so to our knowledge Theorem 3.5 below is new even when the righthand side is Carathéodory.

Definition 3.3. We say that $x \in W_{o}^{1, p}(Z)$ is a solution of type I if there exists some $w \in L^{q^{*}}(Z)$ with $w(z) \in\left[f_{1}(z, x(z)), f_{2}(z, x(z))\right]$ such that

$$
-\operatorname{div}\left(\|D x(z)\|^{p-2} D x(z)\right)-\lambda_{1}|x(z)|^{p-2} x(z)=w(z) \quad \text { a.e. on } Z \text {. }
$$

Definition 3.4. We say that $x \in W_{o}^{1, p}(Z)$ is a solution of type II if $x$ satisfies

$$
-\operatorname{div}\left(\|D x(z)\|^{p-2} D x(z)\right)-\lambda_{1}|x(z)|^{p-2} x(z)=f(z, x(z)) \quad \text { a.e. on } Z \text {. }
$$

It is well known that the existence of a solution of type I does not imply the existence of type II.

First, we derive an existence result of type I and then, using a stronger set of hypotheses, we obtain an existence result of type II.

THEOREM 3.5. If Hypothesis 3.1 holds, then problem (1.1) has a nontrivial solution of type I.

Proof. Let $R_{1}: W_{o}^{1, p}(Z) \rightarrow \mathbb{R}$ such that $R_{1}(x)=(1 / p)\left\|D x_{n}\right\|_{p}^{p}-\left(\lambda_{1} / p\right)\left\|x_{n}\right\|_{p}^{p}$, and $R_{2}: W_{o}^{1, p}(Z) \rightarrow \mathbb{R}$ such that $R_{2}(x)=-\int_{Z} F(z, x(z)) d z$ with $F(z, x)=\int_{o}^{x} f(z, r) d r$. So our energy functional is $R=R_{1}+R_{2}$. It is well known that $R$ is locally Lipschitz (see Chang [3]).

Claim 3.6. The functional $R(\cdot)$ satisfies the $(P S)_{c,+}$-condition in the sense of Costa and Gonçalves [5].

Indeed, let $\left\{x_{n}\right\}_{n \geq 1} \subseteq W_{o}^{1, p}(Z)$ such that $R\left(x_{n}\right) \rightarrow c$ and

$$
R\left(x_{n}\right) \leq R(x)+\varepsilon_{n}\left\|x-x_{n}\right\|, \quad\left\|x-x_{n}\right\| \leq \delta_{n}
$$

with $\varepsilon_{n}, \delta_{n} \rightarrow 0$. 
Let $x=x_{n}+\delta x_{n}$ with $\delta\left\|x_{n}\right\| \leq \delta_{n}$. Divide with $\delta$.

It is easy to see that

$$
\lim _{\delta \downarrow 0} \frac{R_{1}\left(x_{n}+\delta x_{n}\right)-R_{1}\left(x_{n}\right)}{\delta}=\left\|D x_{n}\right\|_{p}^{p}-\lambda_{1}\left\|x_{n}\right\|_{p}^{p} .
$$

Moreover, we have

$$
\lim _{\delta \downarrow 0} \frac{R_{2}\left(x_{n}+\delta x_{n}\right)-R_{2}\left(x_{n}\right)}{\delta} \leq R_{2}^{o}\left(x_{n} ; x_{n}\right) .
$$

Thus,

$$
R_{2}^{o}\left(x_{n} ; x_{n}\right)+\left\|D x_{n}\right\|_{p}^{p}-\lambda_{1}\left\|x_{n}\right\|_{p}^{p} \geq-\varepsilon_{n}\left\|x_{n}\right\| .
$$

On the other hand, for the (PS) $)_{c,-}$ we have

$$
\mathbb{R}(x) \leq \mathbb{R}\left(x_{n}\right)+\varepsilon_{n}\left\|x-x_{n}\right\|, \quad\left\|x-x_{n}\right\| \leq \delta_{n},
$$

with $\varepsilon_{n}, \delta_{n} \rightarrow 0$. Equation (3.8) is equivalent to

$$
(-R)(x)-(-R)\left(x_{n}\right) \geq-\varepsilon_{n}\left\|x-x_{n}\right\|, \quad\left\|x-x_{n}\right\| \leq \delta_{n},
$$

with $\varepsilon_{n}, \delta_{n} \rightarrow 0$. Note that $(-R)$ is locally Lipschitz too.

Choose here $x=x_{n}-\delta x_{n}$. Then as before we have that

$$
\begin{aligned}
& \lim _{\delta \downarrow 0} \frac{\left(-R_{1}\right)\left(x_{n}-\delta x_{n}\right)-\left(-R_{1}\right)\left(x_{n}\right)}{\delta}=\left\|D x_{n}\right\|_{p}^{p}-\lambda_{1}\left\|x_{n}\right\|_{p}^{p}, \\
& \lim _{\delta \downarrow 0} \frac{\left(-R_{2}\right)\left(x_{n}-\delta x_{n}\right)-\left(-R_{2}\right)\left(x_{n}\right)}{\delta} \leq\left(-R_{2}\right)^{o}\left(x_{n} ;-x_{n}\right)=R_{2}^{o}\left(x_{n} ; x_{n}\right) .
\end{aligned}
$$

Thus, finally we obtain again (3.7).

Note that there exists some $w_{n}^{\prime} \in \partial\left(R_{2}\left(x_{n}\right)\right)$ such that $\left\langle w_{n}^{\prime}, x_{n}\right\rangle=R_{2}^{o}\left(x_{n} ; x_{n}\right)$. This means that

$$
\left\langle w_{n}, x_{n}\right\rangle-\left\|D x_{n}\right\|_{p}^{p}+\lambda_{1}\left\|x_{n}\right\|_{p}^{p} \leq \varepsilon_{n}\left\|x_{n}\right\|
$$

for some $w_{n} \in \partial\left(-R_{2}\left(x_{n}\right)\right)$. Note that $w_{n}(z) \in\left[f_{1}\left(z, x_{n}(z)\right), f_{2}\left(z, x_{n}(z)\right)\right]$. 
From the choice of the sequence $\left\{x_{n}\right\} \subseteq W_{o}^{1, p}(Z)$, we have

$$
\theta R\left(x_{n}\right) \leq M_{1} \quad \text { for some } M_{1}>0
$$

Adding (3.11) and (3.12), we have

$$
\begin{gathered}
\left(\frac{\theta}{p}-1\right)\left\|D x_{n}\right\|_{p}^{p}+\lambda_{1}\left(1-\frac{\theta}{p}\right)\left\|x_{n}\right\|_{p}^{p}+\int_{Z}\left(w_{n}(z) x_{n}(z)-\theta F\left(z, x_{n}(z)\right)\right) d z \\
\leq \varepsilon_{n}\left\|x_{n}\right\|+M_{1} .
\end{gathered}
$$

From Hypothesis 3.1(ii) we know that for almost all $z \in Z$ and all $x \in \mathbb{R}$, we have $v x-\theta F(z, x)+a(z) \geq 0$ for some $a \in L^{q^{*}}(Z)$ and for every $v \in \partial(F(z, x))$.

Suppose now that $\left\|x_{n}\right\| \rightarrow \infty$. Inequality (3.13) becomes then

$$
\begin{aligned}
\left(\frac{\theta}{p}-1\right)\left\|D x_{n}\right\|_{p}^{p}+\lambda_{1}\left(1-\frac{\theta}{p}\right)\left\|x_{n}\right\|_{p}^{p} & \\
+\int_{Z}\left(w_{n}(z) x_{n}(z)\right. & \left.-\theta F\left(z, x_{n}(z)\right)\right) d z+\int_{Z} a(z) d z \\
& \leq \varepsilon_{n}\left\|x_{n}\right\|+\int_{Z} a(z) d z+M_{1} .
\end{aligned}
$$

Divide this inequality with $\left\|D x_{n}\right\|_{p}^{p}$, then we have in the limit

$$
\frac{\theta}{p}-1 \leq 0
$$

recall that $\left\|D x_{n}\right\|$ is an equivalent norm in $W_{o}^{1, p}(Z)$ and

$$
-\lambda_{1}\left(1-\frac{\theta}{p}\right)\left\|x_{n}\right\|_{p}^{p} \geq-\left(\frac{\theta}{p}-1\right)\left\|D x_{n}\right\|_{p}^{p}
$$

Since $\theta>p$, we have a contradiction. So $\left\|x_{n}\right\|$ is bounded.

From the properties of the subdifferential of Clarke, we have

$$
\begin{aligned}
\partial R\left(x_{n}\right) & \subseteq \partial\left(R_{1}\left(x_{n}\right)\right)+\partial\left(R_{2}\left(x_{n}\right)\right) \\
& \subseteq \partial\left(R_{2}\left(x_{n}\right)\right)+\partial\left(\frac{1}{p}\left\|D x_{n}\right\|_{p}^{p}-\frac{\lambda_{1}}{p}\left\|x_{n}\right\|_{p}^{p}\right)
\end{aligned}
$$

(see Clarke [4, page 83]). So, we have

$$
\left\langle w_{n}, y\right\rangle=\left\langle A x_{n}, y\right\rangle-\int_{Z} v_{n}(z) y(z) d z
$$


with $w_{n}$ the element with minimal norm of the subdifferential of $R$ (recall that $\left.\left\|w_{n}\right\|_{*} \rightarrow 0\right), v_{n} \in\left[f_{1}\left(z, x_{n}(z)\right), f_{2}\left(z, x_{n}(z)\right)\right]$, and $A: W_{o}^{1, p}(Z) \rightarrow W^{-1, q}(Z)$ such that

$$
\langle A x, y\rangle=\int_{Z}\|D x(z)\|^{p-2}(D x(z), D y(z))_{R^{N}} d z-\lambda_{1} \int_{Z}\left\|x_{n}\right\|_{p}^{p-2} x_{n} y_{n} d z
$$

for all $y \in W_{o}^{1, p}(Z)$. But $x_{n} \stackrel{w}{\rightarrow} x$ in $W_{o}^{1, p}(Z)$, so $x_{n} \rightarrow x$ in $L^{p}(Z)$ and $x_{n}(z) \rightarrow x(z)$ a.e. on $Z$ by virtue of the compact embedding $W_{o}^{1, p}(Z) \subseteq L^{p}(Z)$. Note that $v_{n}$ is bounded. Choose $y=x_{n}-x$. Then in the limit we have that $\lim \sup \left\langle A x_{n}, x_{n}-\right.$ $x\rangle=0$. Recall the following inequality:

$$
\sum_{j=1}^{N}\left(a_{j}(\eta)-a_{j}\left(\eta^{\prime}\right)\right)\left(\eta_{j}-\eta_{j}^{\prime}\right) \geq C\left|\eta-\eta^{\prime}\right|^{p},
$$

for $\eta, \eta^{\prime} \in R^{N}$, with $a_{j}(\eta)=|\eta|^{p-2} \eta_{j}$.

By virtue of this inequality we have that $D x_{n} \rightarrow D x$ in $L^{p}(Z)$. So we have $x_{n} \rightarrow x$ in $W_{o}^{1, p}(Z)$. The claim is proved. Thus $R$ satisfies $(\mathrm{PS})_{c}$.

We will show now that there exists $\rho>0$ such that $R(x) \geq \eta>0$ with $\|x\|=\rho$. To this end, we show that for every sequence $\left\{x_{n}\right\}_{n \geq 1} \subseteq W_{o}^{1, p}(Z)$ with $\left\|x_{n}\right\|=$ $\rho_{n} \rightarrow 0$, we have $R\left(x_{n}\right) \downarrow 0$. Suppose that it is not true. Then there exists a sequence as above such that $R\left(x_{n}\right) \leq 0$. Since $\left\|x_{n}\right\| \rightarrow 0$ we have $x_{n}(z) \rightarrow 0$ a.e. on $Z$.

So we have

$$
\left\|D x_{n}\right\|_{p}^{p}-\lambda_{1}\left\|x_{n}\right\|_{p}^{p} \leq \int_{Z} p F\left(z, x_{n}(z)\right) d z
$$

Let $y_{n}(z)=x_{n}(z) /\left\|x_{n}\right\|_{1, p}$. Also, from Hypothesis 3.1(iii) we have uniformly, for all $z \in Z$, that for all $\varepsilon>0$ we can find $\delta>0$ such that for $|x| \leq \delta$ we have

$$
p F(z, x(z)) \leq \theta(z)|x(z)|^{p}+\varepsilon|x(z)|^{p} .
$$

On the other hand, from hypothesis (i) we have that there exist some $c_{1}, c_{2}$ such that $p F(z, x) \leq c_{1}|x|^{p}+c_{2}|x|^{p^{*}}+p|x|$ for almost all $z \in Z$ and all $x \in \mathbb{R}$. Thus we can always find some $\gamma>0$ such that $p F(z, x) \leq(\theta(z)+\varepsilon)|x| p+\gamma|x| p^{*}$. Indeed, choose $\gamma \geq\left|c_{1}-\theta(z)-\varepsilon\right||\delta|^{p-p^{*}}+c_{2}+p|\delta|^{1-p^{*}}$.

Then we obtain,

$$
\left\|D x_{n}\right\|_{p}^{p}-\lambda_{1}|| x_{n} \|_{p}^{p} \leq \int_{Z}(\theta(z)+\varepsilon)\left|x_{n}(z)\right|^{p} d z+\gamma \int_{Z}\left|x_{n}(z)\right|^{p^{*}} d z .
$$


Dividing the last inequality, by $\left\|x_{n}\right\|_{1, p}^{p}$, we have

$$
\begin{aligned}
\left\|D y_{n}\right\|^{p}-\lambda_{1}\left\|y_{n}\right\|_{p}^{p} & \leq \int_{Z}(\theta(z)+\varepsilon)\left|y_{n}(z)\right|^{p} d z+\gamma \frac{\int_{Z}\left|x_{n}(z)\right|^{p^{*}} d z}{\left\|x_{n}\right\|_{1, p}^{p}} \\
& \leq \varepsilon\left\|y_{n}\right\|_{p}^{p}+\gamma_{1}\left\|x_{n}\right\|_{1, p}^{p^{*}-p},
\end{aligned}
$$

recall that $W_{o}^{1, p}(Z)$ is continuously embedded on $L^{p^{*}}(Z)$.

Using the variational characterization of the first eigenvalue we have that $0 \leq$ $\left\|D y_{n}\right\|_{p}^{p}-\lambda_{1}\left\|y_{n}\right\|_{p}^{p} \leq \varepsilon\left\|y_{n}\right\|_{p}^{p}+\gamma_{1}\left\|x_{n}\right\|_{1, p}^{p^{*}-p}$.

Recall that $\left\|y_{n}\right\|=1$ so $y_{n} \rightarrow y$ weakly in $W_{o}^{1, p}(Z), y_{n}(z) \rightarrow y(z)$ a.e. on $Z$. Thus, from (3.24) we have that $\left\|D y_{n}\right\| \rightarrow \lambda_{1}\|y\|$. Also, from the weak lower semicontinuity of the norm we have that $\|D y\| \leq \liminf \left\|D y_{n}\right\| \rightarrow \lambda_{1}\|y\|$. Using the Rayleigh quotient we have that $\|D y\|=\lambda_{1}\|y\|$. Recall that $y_{n} \rightarrow y$ weakly in $W_{o}^{1, p}(Z)$ and $\left\|D y_{n}\right\| \rightarrow\|D y\|$. So, from a well-known argument we obtain $y_{n} \rightarrow y$ in $W_{o}^{1, p}(Z)$, and since $\left\|y_{n}\right\|=1$ we have that $\|y\|=1$. That is, $y \neq 0$ and from the equality $\|D y\|=\lambda_{1}\|y\|$ we have that $y(z)= \pm u_{1}(z)$. Suppose that $y(z)=u_{1}(z)$.

Dividing now (3.23) by $\left\|x_{n}\right\|_{1, p}^{p}$ and using the variational characterization of the first eigenvalue, there exists for every $\varepsilon>0$ some $n_{o}$ such that for $n \geq n_{o}$ we have

$$
0 \leq \int_{Z}(\theta(z)+\varepsilon)\left|y_{n}(z)\right|^{p} d z+\gamma_{1}|| x_{n} \|_{1, p}^{p^{*}-p}
$$

So in the limit we obtain

$$
0 \leq \int_{Z}(\theta(z)+\varepsilon) u_{1}^{p}(z) d z \leq \varepsilon\left\|u_{1}\right\|_{p}^{p} \quad \forall \varepsilon>0 .
$$

Thus, $\int_{Z} \theta(z) u_{1}^{p}(z) d z=0$. Recall that $u_{1}(z)>0$ a.e. on $Z$. This is a contradiction. So there exists $\rho>0$ such that $R(x) \geq \eta>0$ for all $x \in W_{o}^{1, p}(Z)$ with $\|x\|=\rho$.

Next, it is easy to see that

$$
R\left(s u_{1}\right)=-\int_{Z} F\left(z, s u_{1}(z)\right) d z
$$

(here we used again the Rayleigh quotient).

But from hypothesis (ii) we have that $-F\left(z, s u_{1}(z)\right) \leq-c_{3}\left|s u_{1}(z)\right|^{\theta}+a_{1}(z)$ a.e. on $Z$. So for $s$ large enough, we obtain that $R\left(s u_{1}\right) \leq 0$. Then we can use Theorem 2.2 to obtain $x \in W_{o}^{1, p}(Z)$ such that $x \neq 0$ and $0 \in \partial R(x)$. It follows that

$$
A x=\lambda_{1}|x|^{p-2} x+v
$$


with $v \in \partial\left(\int_{Z} F(z, x(z)) d z\right)$. So for every $\phi \in C_{o}^{\infty}(Z)$ we have

$$
\langle A x, \phi\rangle=\lambda_{1}\left\langle|x|^{p-2} x, \phi\right\rangle_{p q}+(\nu, \phi)_{p q} .
$$

By $(\cdot, \cdot)_{p q}$ we denote the duality brackets for the pair $\left(L^{p}(Z), L^{q}(Z)\right)$. Thus,

$$
\int_{Z}\|D x(z)\|^{p-2}(D x(z), D \phi(z))_{R^{N}} d z=\int_{Z}\left(\lambda_{1}|x(z)|^{p-2} x(z)+v(z)\right) \phi(z) d z .
$$

From the definition of the distributional derivative,

$$
-\operatorname{div}\left(\| D x(z)||^{p-2} D x(z)\right)-\lambda_{1}|x(z)|^{p-2} x(z)=v(z) \quad \text { a.e. on } Z \text {. }
$$

So $x \in W_{o}^{1, p}(Z)$ is a nontrivial solution of type I.

In order to have an existence result of type II, we have to impose stronger hypotheses on $f$. Our hypotheses are the following.

Hypothesis 3.7. The function $f: Z \times \mathbb{R} \rightarrow \mathbb{R}$ satisfies Hypothesis 3.1. Moreover, we suppose that $f_{1}(z, a) d z+\lambda_{1}|a|^{p-2} a>0$ or that $f_{2}(z, a)+\lambda_{1}|a|^{p-2} a<0$ a.e. on $Z$, for any $a \in D(f)=\left\{x \in \mathbb{R}: f_{1}(z, x) \neq f_{2}(z, x)\right.$ a.e. on $\left.S_{x} \subseteq Z\right\}$ (i.e., the set of the discontinuity points of $f)$. Finally, we suppose that $f(z, \cdot)$ has countable number of discontinuities.

THEOREM 3.8. If Hypothesis 3.7 holds, then problem (1.1) has a nontrivial solution of type II.

Proof. From Theorem 3.5 we know that there exists a nontrivial solution of type I. That is, there exists some $w \in L^{q}(Z)$ with $w(z) \in\left[f_{1}(z, x(z)), f_{2}(z, x(z))\right]$ such that

$$
\begin{gathered}
-\operatorname{div}\left(\|D x(z)\|^{p-2} D x(z)\right)-\lambda_{1}|x(z)|^{p-2} x(z)=w(z) \quad \text { a.e. on } Z, \\
\left.x\right|_{\Gamma}=0 .
\end{gathered}
$$

We suppose that there exists some $A \subseteq Z$ with $|A|>0$ such that $x(z)=a_{1} \in$ $D(f)$ a.e. on $A$, and that $\left|A \cap S_{a_{1}}\right| \neq 0$. Take now the closure of that set, that is, $\overline{A \cap S_{a_{1}}}$. It is clear that the interior of that set is nonempty (recall that $\overline{A \cap S_{a_{1}}}=$ $\left.\left(A \cap S_{a_{1}}\right)^{o} \cup \partial\left(A \cap S_{a_{1}}\right)\right)$ because we have supposed that $\left|A \cap S_{a_{1}}\right| \neq 0$. So, there exist some $z \in\left(A \cap S_{a_{1}}\right)^{o}$ and some $r>0$ such that $B(z, r) \subseteq A \cap S_{a_{1}}$. Take now $r^{\prime}=r / 2$, then it is clear that $B\left(z, r^{\prime}\right) \subseteq B(z, r) \subseteq A \cap S_{a_{1}}$ (here by $B(z, r)$ we denote the open ball centered at $z$ with radius $r$ ).

We know that there exists a test function which is equal to 1 on $\overline{B\left(z, r^{\prime}\right)}$, equal to 0 outside $B(z, r)$, and assumes values in $[0,1]$ in $B(z, r) \backslash \overline{B\left(z, r^{\prime}\right)}$. Multiply (3.32) with this function and then integrate over $B(z, r)$. Using the definition of the distributional derivative and finally the well-known theorem of Stampacchia, 
which states that if $x(z) \in W^{1, p}(Z)$ and $x(z)=a$ a.e. on $A$ then $D_{k} x(z)=0$ a.e. on $A$, we have

$$
\int_{B(z, r)} w(z) \phi(z) d z=\int_{B(z, r)}\left(-\lambda_{1}\left|a_{1}\right|^{p-2} a_{1}\right) \phi(z) d z
$$

But we know that $w(z) \in\left[f_{1}(z, x(z)), f_{2}(z, x(z))\right]$ a.e. on $\mathrm{Z}$.

If $f_{1}\left(z, a_{1}\right)+\lambda_{1}\left|a_{1}\right|^{p-2} a_{1}>0$ a.e. on $Z$, we obtain

$$
\begin{aligned}
0 & <\int_{B(z, r)}\left(f_{1}\left(z, a_{1}\right)+\lambda_{1}\left|a_{1}\right|^{p-2} a_{1}\right) \phi(z) d z \\
& \leq \int_{B(z, r)}\left(w(z)+\lambda_{1}\left|a_{1}\right|^{p-2} a_{1}\right) \phi(z) d z=0 .
\end{aligned}
$$

Thus we have a contradiction. The same holds if $f_{2}\left(z, a_{1}\right) d z+\lambda_{1}\left|a_{1}\right|^{p-2} a_{1}<0$ a.e. on $Z$. So $\left|A \cap S_{a_{1}}\right|=0$. Set now $B \subseteq Z$ such that

$$
B=\bigcup_{n=1}^{\infty} B_{n},
$$

where $B_{n}=A_{n} \cap S_{a_{n}} \subseteq Z$ is such that $x(z)=a_{n}$ on $A_{n}$ with $a_{n} \in D(f)$ (recall that $f$ has countable number of discontinuities). Then from the above arguments we have that $|B|=0$. That is, $x$ is a solution of type II.

Remark 3.9. As far as we know, this is the first existence result of type II for the $p$-Laplacian with nonmonotone discontinuities and without using the method of upper and lower solution. All the known results need the solution to be in $W_{o}^{2, p}(Z)$ (cf. [1]), but here we do not have such a regularity result, so the arguments that we have used are more complicated.

\section{Acknowledgment}

This work is partially supported by a postdoctoral scholarship from the State Scholarship Foundation (IKY) of Greece.

\section{References}

[1] A. Ambrosetti and M. Badiale, The dual variational principle and elliptic problems with discontinuous nonlinearities, J. Math. Anal. Appl. 140 (1989), no. 2, 363-373.

[2] J. Bouchala and P. Drábek, Strong resonance for some quasilinear elliptic equations, J. Math. Anal. Appl. 245 (2000), no. 1, 7-19.

[3] K. C. Chang, Variational methods for nondifferentiable functionals and their applications to partial differential equations, J. Math. Anal. Appl. 80 (1981), no. 1, 102129.

[4] F. H. Clarke, Optimization and Nonsmooth Analysis, John Wiley \& Sons, New York, 1983.

[5] D. G. Costa and J. V. A. Gonçalves, Critical point theory for nondifferentiable functionals and applications, J. Math. Anal. Appl. 153 (1990), no. 2, 470-485. 
[6] P. Lindqvist, On the equation $\operatorname{div}\left(|\nabla u|^{p-2} \nabla u\right)+\lambda|u|^{p-2} u=0$, Proc. Amer. Math. Soc. 109 (1990), no. 1, 157-164.

[7] D. Motreanu and P. D. Panagiotopoulos, A minimax approach to the eigenvalue problem of hemivariational inequalities and applications, Appl. Anal. 58 (1995), no. 1-2, $53-76$.

Halidias Nikolaos: Department of Statistics and Actuarial Science, School of Sciences, University of the Aegean, Karlovassi, 83200 Samos, Greece

E-mail address: nick@aegean.gr 


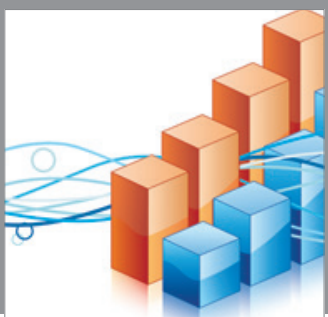

Advances in

Operations Research

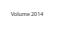

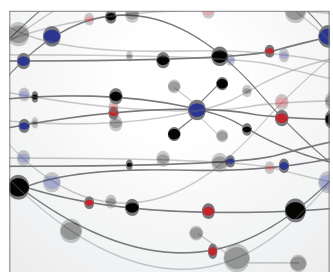

\section{The Scientific} World Journal
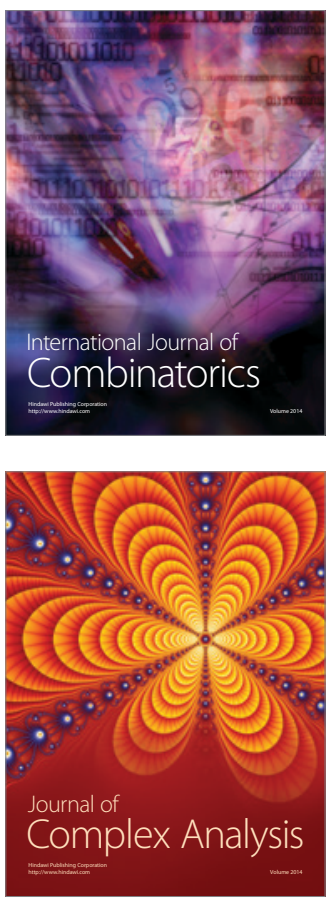

International Journal of

Mathematics and

Mathematical

Sciences
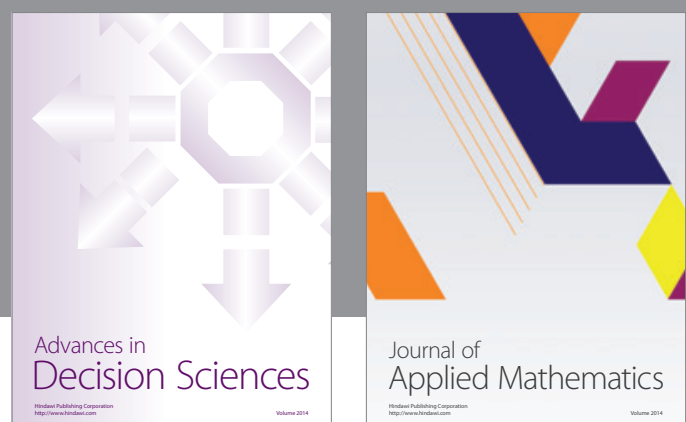

Journal of

Applied Mathematics
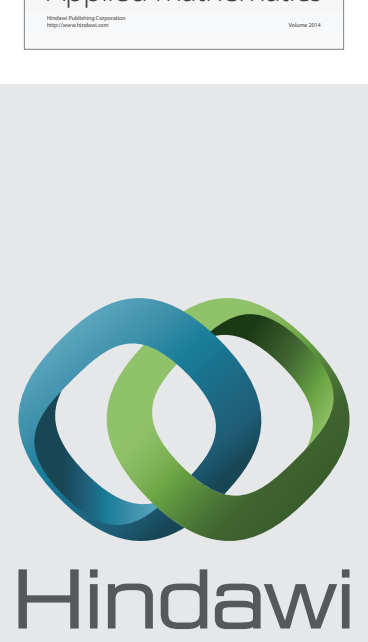

Submit your manuscripts at http://www.hindawi.com
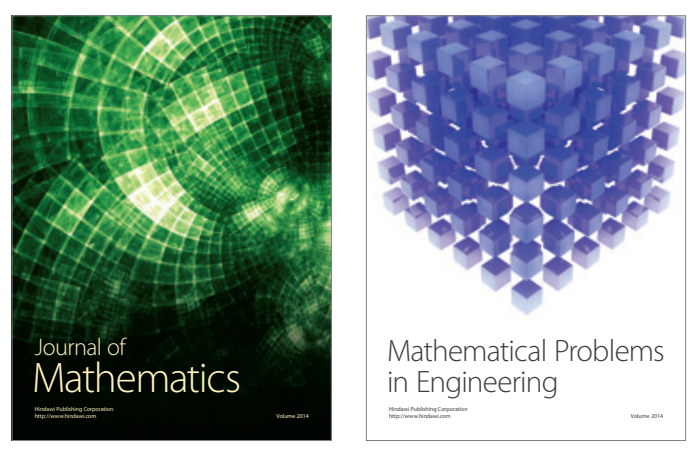

Mathematical Problems in Engineering
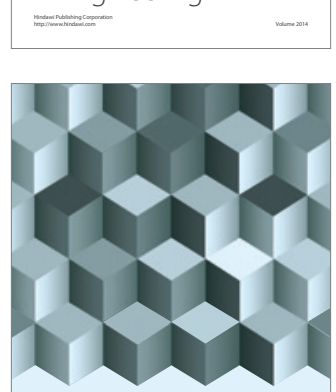

Journal of

Function Spaces
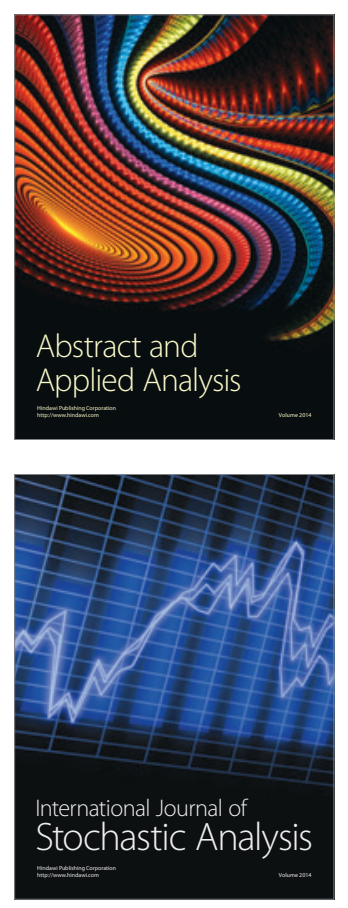

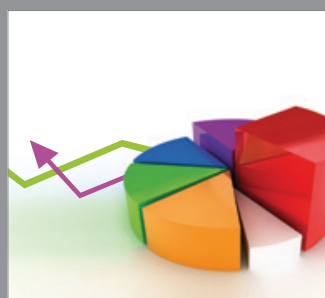

ournal of

Probability and Statistics

Promensencen
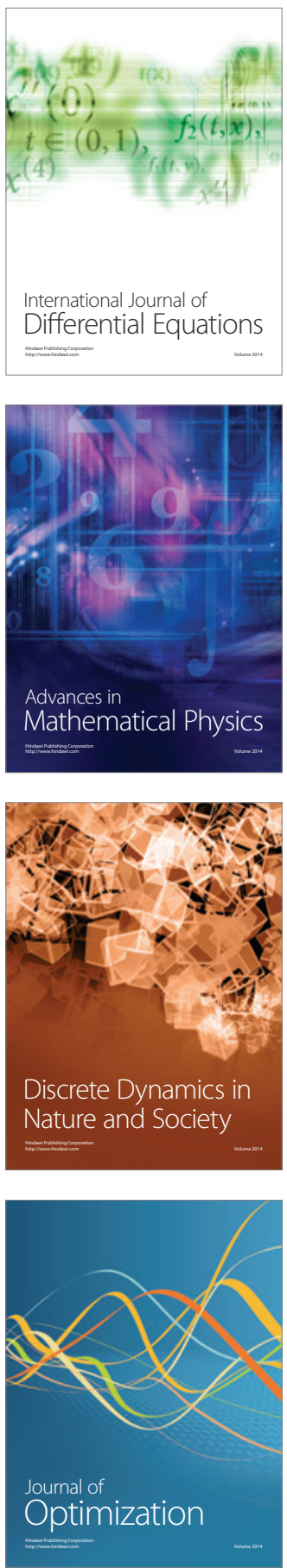